\title{
CRANIOFARINGIOMAS \\ ACHADOS CLÍNICOS, EPIDEMIOLÓGICOS E ANATOMOPATOLÓGICOS DE 25 CASOS
}

\author{
LUIZ FERNANDO BLEGGI TORRES*, JORGE SERGIO REIS FILHO**, \\ MÁRIO R. MONTEMÓR NETTO**, LINEI A. B. DELLÉ***, BEATRIZ GARCIA SLUMINSKY***, \\ LEONARDO N. FAORO***, AFONSO ANTONIUK****, RICARDO RAMINA*****
}

\begin{abstract}
RESUMO - Relatamos os achados clínicos, epidemiológicos e anatomopatológicos de 25 casos de craniofaringiomas. Dos 25 pacientes analisados, 14 ocorreram em pacientes do sexo masculino e 11 em pacientes femininos. As idades variaram de 3 a 64 anos, com média de 30,52 anos. Quanto à localização, 12 casos foram supra-selares, 10 ocorreram em região selar, sendo que 8 apresentaram extensão supra-selar, dois localizaram-se em lobo frontal e 1 em região ponto-cerebelar. Os sinais e sintomas observados incluiram alterações visuais ( $72 \%$ dos pacientes), cefaléia (68\%), vômitos (40\%) e edema de papila (24\%), com período de evolução variando de 18 dias a 60 meses, com média de 11,66 meses. Treze pacientes foram submetidos a ressecção cirúrgica parcial, 11 a exérese total e 1 a ressecção parcial associada a quimioterapia adjuvante. Evidenciou-se taxa de recidiva tumoral total de $48 \%$. Sete recidivas tumorais ocorreram nos pacientes submetidos apenas a exérese parcial, quatro nos pacientes submetidos a tumorectomia total e 1 no submetido a tratamento cirúrgico associado a quimioterapia. Estes achados aproximam-se dos encontrados na literatura, corroborando para a compreensão do comportamento biológico deste tumor e apontando a extensão do tumor residual como único fator prognóstico para recidiva desta neoplasia.
\end{abstract}

PALAVRAS-CHAVE: craniofaringiomas, tumores do sistema nervoso central, neoplasias cerebrais.

\section{Craniopharyngiomas: clinical, epidemiological and pathological findings of $\mathbf{2 5}$ cases}

ABSTRACT - We report the clinical and pathological findings of 25 cases of craniopharyngiomas. Fourteen patients were males and 11 were females. The ages ranged between 3 and 64 years, with a mean of 30.52 years. The symptoms reflected the growth and topography of the tumours; visual disorders (72\%), headache (68\%), vomits (40\%) and papilledema (24\%) were the most frequent symptoms. Twelve cases were suprasellar; 10 tumours arose from sellar region, from which 8 presented suprasellar extension; frontal lobe $(n=2)$ and ponto cererebellar angle $(n=1)$ were also affected. Eleven patients undergone total resection of the tumor and 14 were submitted to partial resection, from which 1 received adjuvant chemotherapy. The overall recurrence rate was $48 \%$. Eight recurrences were observed in the patients submitted to partial resection and 4 in which total resection were performed. These results are similar with the literature, corroborating to the extension of residual tumour after the surgical resection as the main prognostic factor for this neoplasm.

KEY WORDS: craniopharyngiomas, central nervous system tumours, brain neoplasms.

Os craniofaringiomas são tumores encapsulados, de origem epitelial, derivados do epitélio da bolsa de Rathke ${ }^{1-4}$, caracterizados por acometerem crianças e adultos jovens, representando a neoplasia intra-craniana não neuroepitelial mais frequente neste grupo etário ${ }^{1}$. Relatamos os achados anatomopatológicos e clínico prognósticos de 25 casos de craniofaringiomas diagnosticados em paci-

Estudo realizado na Seção de Microscopia Eletrônica e Neuropatologia do Serviço de Anatomia Patológica (SAP), Hospital de Clínicas (HC) da Universidade Federal do Paraná (UFPR), Curitiba, PR: *PhD em Neuropatologia (Londres), Chefe do SAP - HC - UFPR; **Médico Residente do SAP - HC - UFPR; ***Estagiário da Seção de Microscopia Eletrônica e Neuropatologia SAP - HC- UFPR; ****Professor Titular da Disciplina de Neurocirurgia UFPR; *****Médico Neurocirurgião do Hospital das Nações - Curitiba. Aceite: 17-dezembro-1998.

Dr. Luiz Fernando Bleggi Torres - Serviço de Anatomia Patológica, Hospital de Clínicas- Rua General Carneiro 181- 80060-900 Curitiba PR - Brasil. Fax 041 2641304. E-Mail: bleggi@hc.ufpr.br e lftorres@uol.com.br 
entes da cidade de Curitiba no período compreendido entre 1990 e 1997, realizando correlação entre o tratamento e o prognóstico dos portadores desta neoplasia. Até o presente momento, este é um dos escassos estudos clínico-patológicos na literatura latino americana indexada no MEDLINE e LILACS.

\section{MÉTODO}

Este estudo deriva de uma linha de pesquisa denominada "Banco de Patologia Tumoral do Sistema Nervoso Central da População da Cidade de Curitiba", que tem como objetivo tabular todos os dados referentes aos tumores de sistema nervoso central (SNC) que acometem a população dessa cidade e sua região metropolitana. A pesquisa tem o intuito de analisar epidemiologicamente os principais tumores do SNC. Para tanto, foram pesquisados os livros de registros ou arquivos de biópsias do período de 1990 a 1997 dos Serviços de Anatomia Patológica dos principais hospitais da Cidade de Curitiba que são responsáveis por mais de $95 \%$ da rotina neurocirúrgica do município. Do total de biópsias de diversos órgãos e tecidos realizadas neste período, foram compiladas as de SNC, pesquisando-se o sexo, idade, localização tumoral e diagnóstico histológico. Deste total, foram separados os craniofaringiomas avaliando-se as variáveis sexo, idade e localização tumoral. Os dados clínicos dos pacientes, como história clínica, tempo de evolução dos sintomas, exame físico, tratamento e evolução, foram obtidos a partir dos prontuários médicos. Todas as biópsias foram preparadas conforme técnicas histológicas convencionais ${ }^{5}$, sendo utilizados os critérios da Classificação da Organização Mundial de Saúde $(\mathrm{OMS})^{1}$ para o diagnóstico dos craniofaringiomas.

\section{RESULTADOS}

Das 3318 biópsias realizadas, $2427(73,14 \%)$ corresponderam a neoplasias primárias do SNC e, dentre estas, $76(3,13 \%)$ tiveram diagnóstico histológico de craniofaringiomas. Destes, 51 não apresentaram dados clínicos prognósticos suficientes para a inclusão no estudo. Dos 25 pacientes analisados, 14 ocorreram em pacientes do sexo masculino e onze em pacientes femininos (Fig 1). As idades variaram de 3 a 64 anos, com média de 30,52 anos (Fig 2). Quanto à localização, 12 casos foram supra-selares, 10 ocorreram na região selar, sendo que 8 apresentaram extensão supra-selar; dois apresentaram acometimento de lobo frontal e 1 localizou-se em região ponto-cerebelar (Fig 3). Os sinais e sintomas observados incluíram alterações visuais (72\% dos pacientes), cefaléia (68\%), vômitos (40\%), edema de papila (24\%), tontura (20\%) e crises convulsivas (16\%), com um período

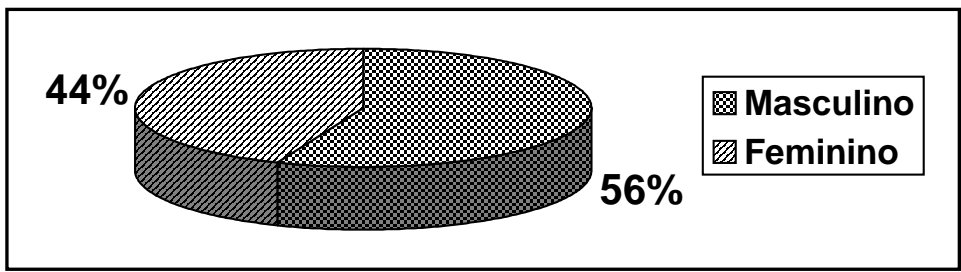

Fig. 1. Distribuição dos pacientes com craniofaringiomas de acordo com o sexo.

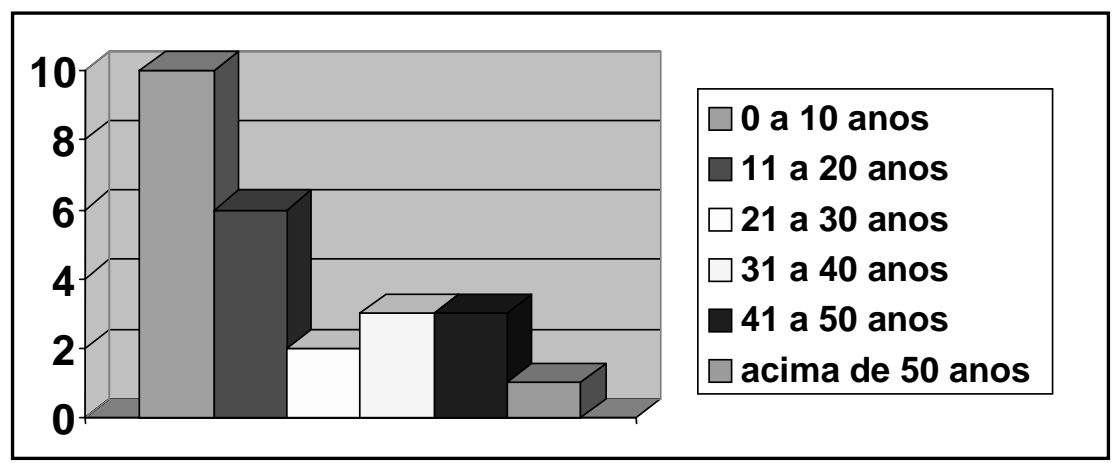

Fig. 2. Histograma mostrando a distribuição etária dos pacientes com craniofaringiomas. 


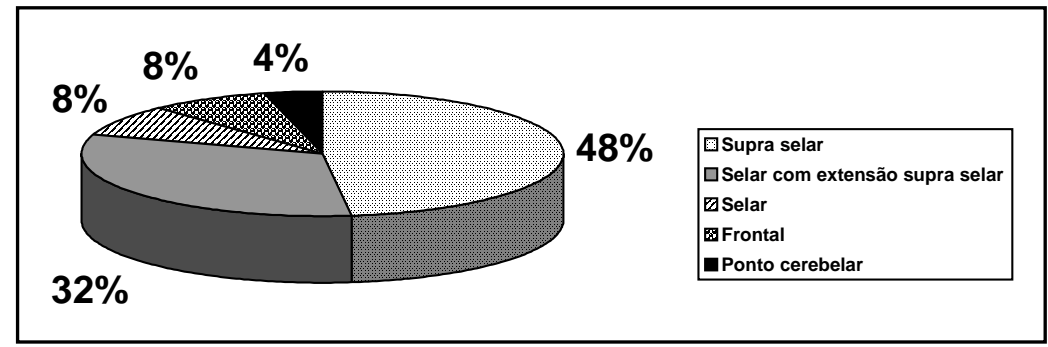

Fig. 3. Localização dos tumores nos pacientes portadores de craniofaringiomas.

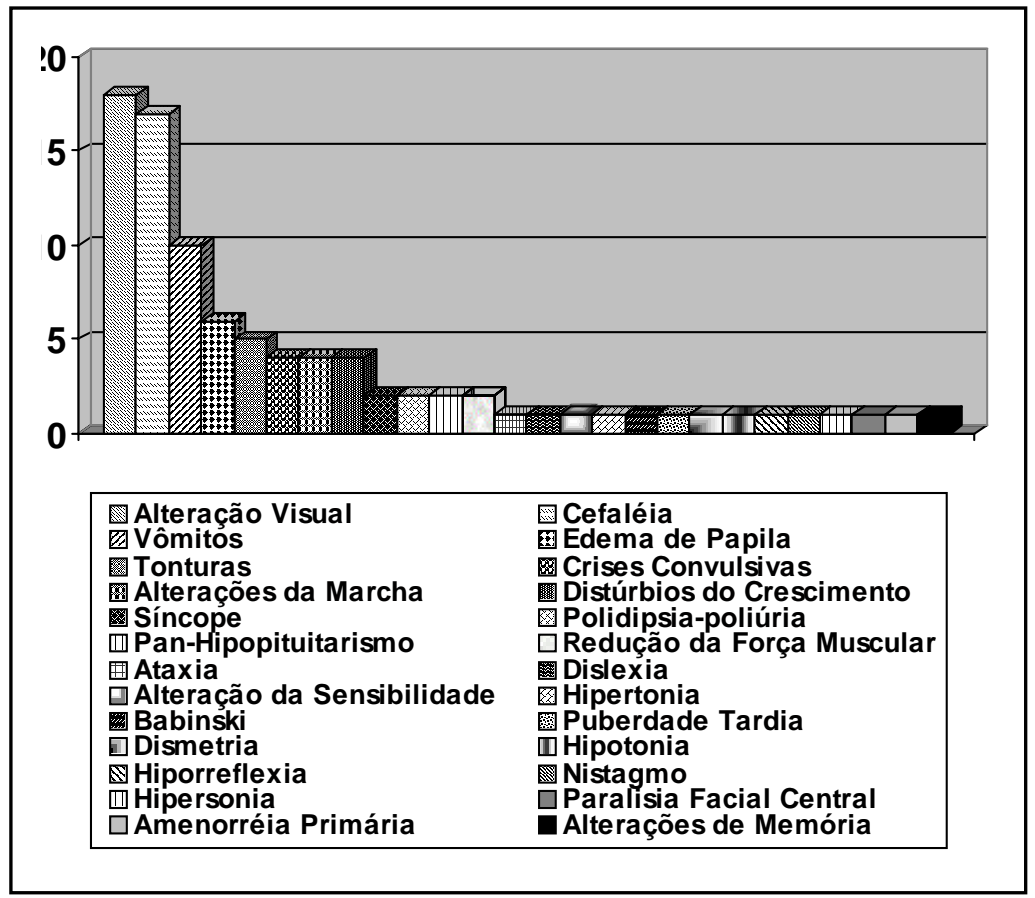

Fig. 4. Sintomatologia apresentada pelos pacientes portadores de craniofaringiomas.

Tabela 1. Taxas de recidiva tumoral nos pacientes portadores de craniofaringiomas.

\begin{tabular}{ccccc}
\hline Tratamento & $\begin{array}{c}\text { Número } \\
\text { de casos }\end{array}$ & $\begin{array}{c}\text { Recidiva } \\
\text { tumoral }\end{array}$ & $\begin{array}{c}\text { Perído médio entre o tratamento } \\
\text { e a recidiva tumoral (meses) }\end{array}$ & $\%$ \\
\hline RT & 11 & 4 & 10,5 & 36,4 \\
RP & 13 & 7 & 19,1 & 53,8 \\
RP + QT & 1 & 1 & 12 & 100 \\
\hline
\end{tabular}

RT, ressecção total; RP, ressecção parcial; QT, quimioterapia. 


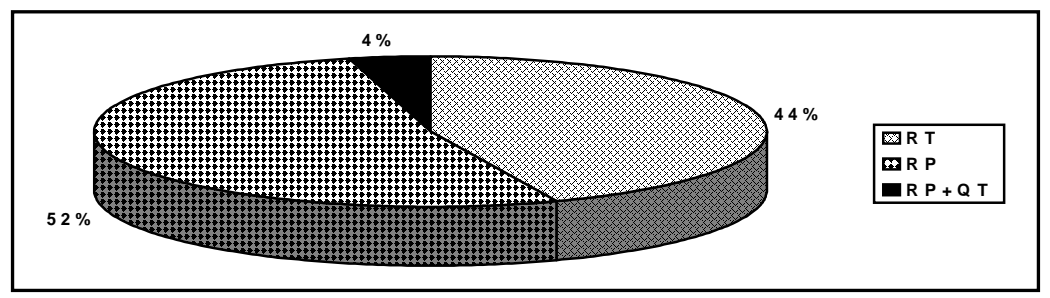

Fig. 5. Tratamento dos pacientes portadores de craniofaringiomas.

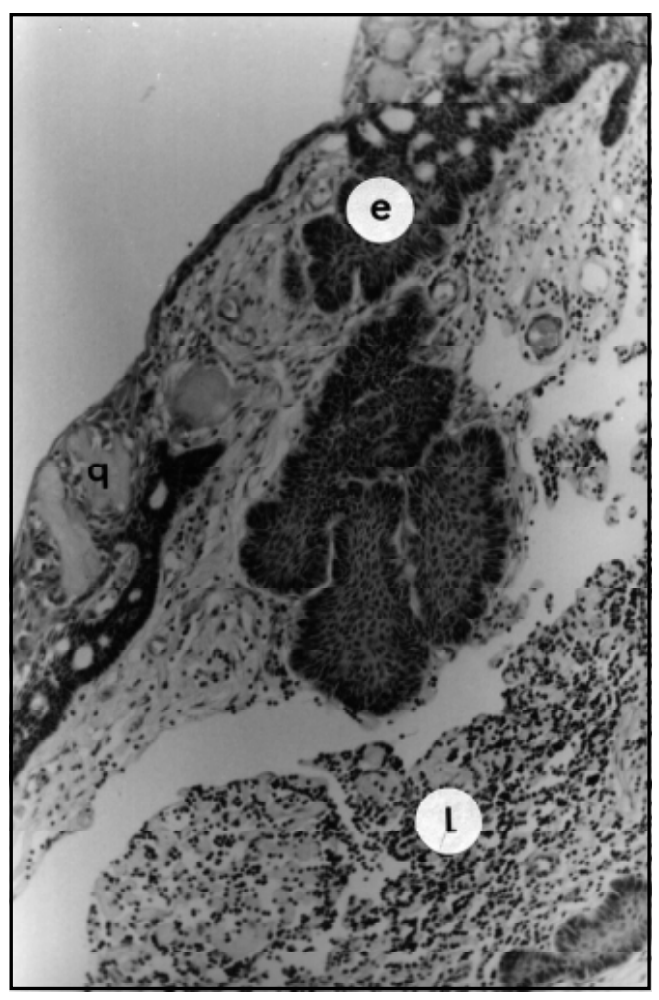

Fig 6. Parede de craniofaringioma com focos de epitélio pavimentoso (e) e áreas queratinizadas (q). Tecido circunjacente com focos de linfócitos (L). HEx100. de evolução variando de 18 dias a 60 meses, com uma média de 11,66 meses (Fig 4). Treze pacientes foram submetidos a ressecção cirúrgica parcial, 11 a exérese total e 1 a ressecção parcial associada a quimioterapia adjuvante (Fig 5), sendo evidenciada uma taxa de recidiva tumoral total de $48 \%$. Sete recidivas tumorais ocorreram nos pacientes submetidos apenas a exérese parcial, com período médio (PM) de 19,1 meses entre o tratamento e a recidiva; 4 nos pacientes submetidos a tumorectomia total $(\mathrm{PM}=10,5$ meses) e 1 no submetido a tratamento cirúrgico associado a quimioterapia (PM=12 meses) (Tabela 1).

\section{DISCUSSÃO}

Os craniofaringiomas correspondem a cerca de 1,2 a $4,6 \%$ de todos os tumores intracranianos, sendo as neoplasias não neuroepiteliais mais frequentes na faixa etária pediátrica $^{6}$, não havendo distinção quanto ao sexo $^{2,7}$. Mais de $50 \%$ dos casos da variante adamantinomatosa acometem pacientes abaixo dos vinte anos de idade, sendo que raros casos de pacientes neonatais apresentando craniofaringiomas foram relatados ${ }^{6}$. A variante papilar acomete quase que exclusivamente pacientes adultos ${ }^{2,7}$, com a moda da incidência situada na quinta década de vida ${ }^{2,7,8}$. Em nosso

estudo, os craniofaringiomas corresponderam a 3,13\% dos tumores primários do SNC, ocorrendo em $54 \%$ dos casos em pacientes do sexo masculino. Apresentaram caráter bimodal, com moda de incidência nas primeiras duas décadas de vida e a outra entre a quarta e a quinta décadas.

Localizam-se predominantemente na região selar, sendo que apenas $5 \%$ dos casos ficam limitados à sela túrcica ${ }^{9}$. Ângulo cerebelo-pontino, nervo óptico, osso esfenóide e faringe constituem sítios anatômicos raramente acometidos ${ }^{9}$. A variante papilar frequentemente compromete o III ventrículo ${ }^{1}$. Em cerca $30 \%$ dos casos observa-se disseminação por contiguidade para regiões anteriores e em $23 \%$ para fossa posterior ${ }^{9}$. Em nossa casuística, $48 \%$ dos tumores apresentaram localização supraselar, $32 \%$ foram selares com extensão supraselar e $8 \%$ foram restritos à sela túrcica. Dois casos de invasão por contiguidade do III ventrículo foram evidenciados. 
A sintomatologia exibida pelos pacientes portadores desta neoplasia está intimamente relacionada com a localização da lesão $0^{6,10}$, sendo os distúrbios visuais, os sintomas mais frequentes em adultos, e as desordens neuro-endócrinas, as mais prevalentes em crianças ${ }^{6,10}$, o que foi corroborado por nosso estudo. Déficit cognitivo e alterações da personalidade podem ocorrer em até $50 \%$ dos casos, principalmente naqueles em que se evidencia invasão do terceiro ventrículo.

Os craniofaringiomas apresentam-se ao estudo anatomopatológico como tumores sólidos, com componente cístico variável; são neoplasias bem delimitadas, com superfície lisa; entretanto podem estar intimamente aderidos a estruturas vasculares e ao tecido cerebral adjacente ${ }^{1-4,6,7}$. Calcificações são achados frequentes na variante adamantinomatosa ${ }^{6}$, o que não se observa na papilar ${ }^{2,7}$. Os cistos da variante adamantinomatosa exibem conteúdo acastanhado, rico em colesterol, com textura similar a óleo de máquina ${ }^{1,6}$, o que não é observado na outra variante ${ }^{2,7}$.

A variante adamantinomatosa (Figs 6 a 8) se caracteriza por cordões ou ninhos de epitélio escamoso multiestratificado, composto por células poligonais ou colunares, dispostas em um arranjo em pseudopaliçada na periferia, enquanto nas regiões centrais evidencia-se textura frouxamente "reticular" 1,6. As paredes dos cistos são compostas por epitélio simples pavimentoso. Grânulos compactos de queratina, apresentando tendência a calcificações distróficas, bem como conteúdo rico em colesterol, fibrose e inflamação crônica reacional constituem importantes elementos diagnósticos ${ }^{1,6}$. A variante papilar não apresenta queratina, nem fluido rico em colesterol, sendo composta por papilas fibrovasculares recobertas por epitélio escamoso multiestratificado ${ }^{2,7}$.

A análise imuno-histoquímica demonstra forte reatividade para queratina de alto peso molecular nas regiões adamantinomatosas e de baixo peso molecular nas papilares ${ }^{11}$. Szeifert et al. ${ }^{12}$ postularam que estes tumores apresentam células neuroendócrinas, o que fora corroborado por estudos imunohistoquímicos demonstrando positividade para cromatogranina A e gonadotrofina coriônica humana ${ }^{11-}$ ${ }^{13}$. O significado da positividade para somatostatina e p-glicoproteína continua incerto ${ }^{11,13}$.

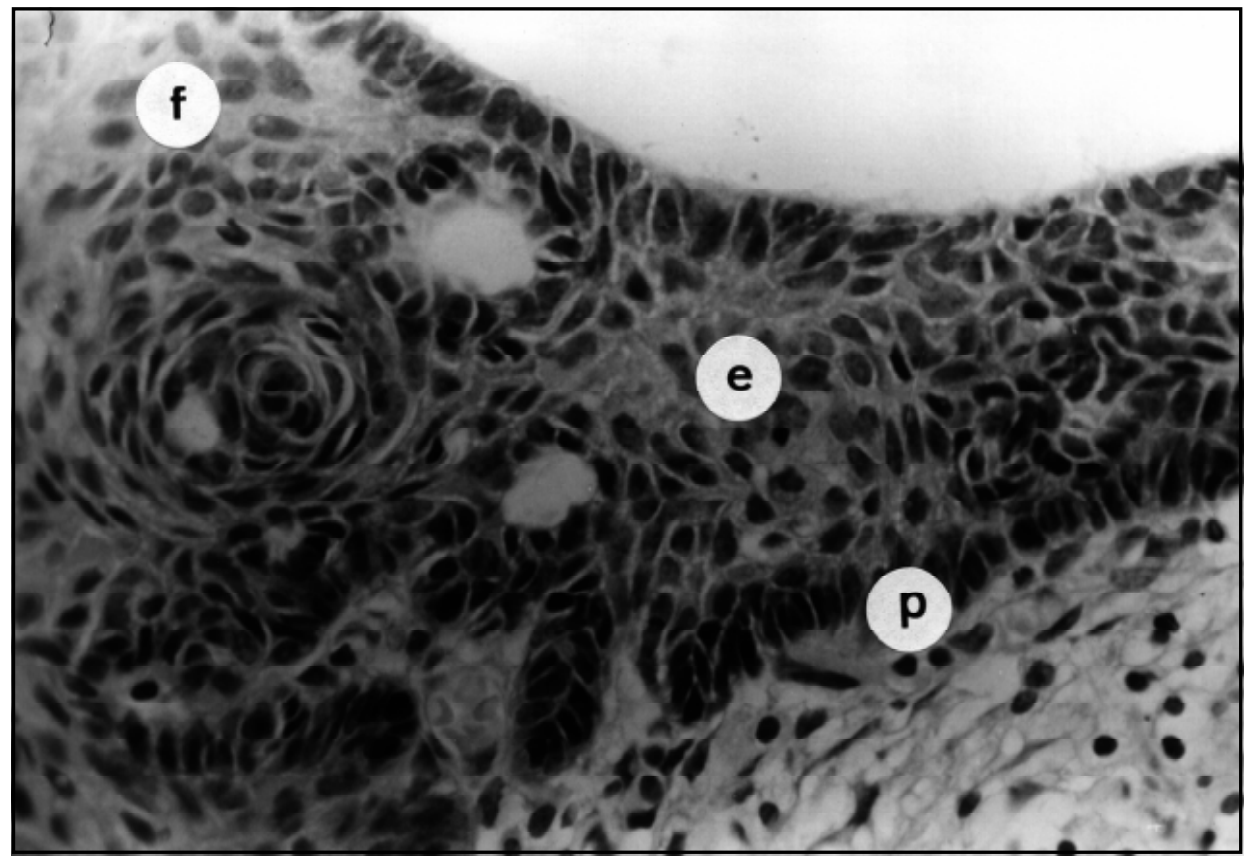

Fig. 7. Detalhe de craniofaringioma tipo adamantinomatoso, composto por ninhos de epitélio pavimentoso (e) com paliçada de células tumorais ( $p$ ) e focos de textura frouxa (f). HE $x 400$. 


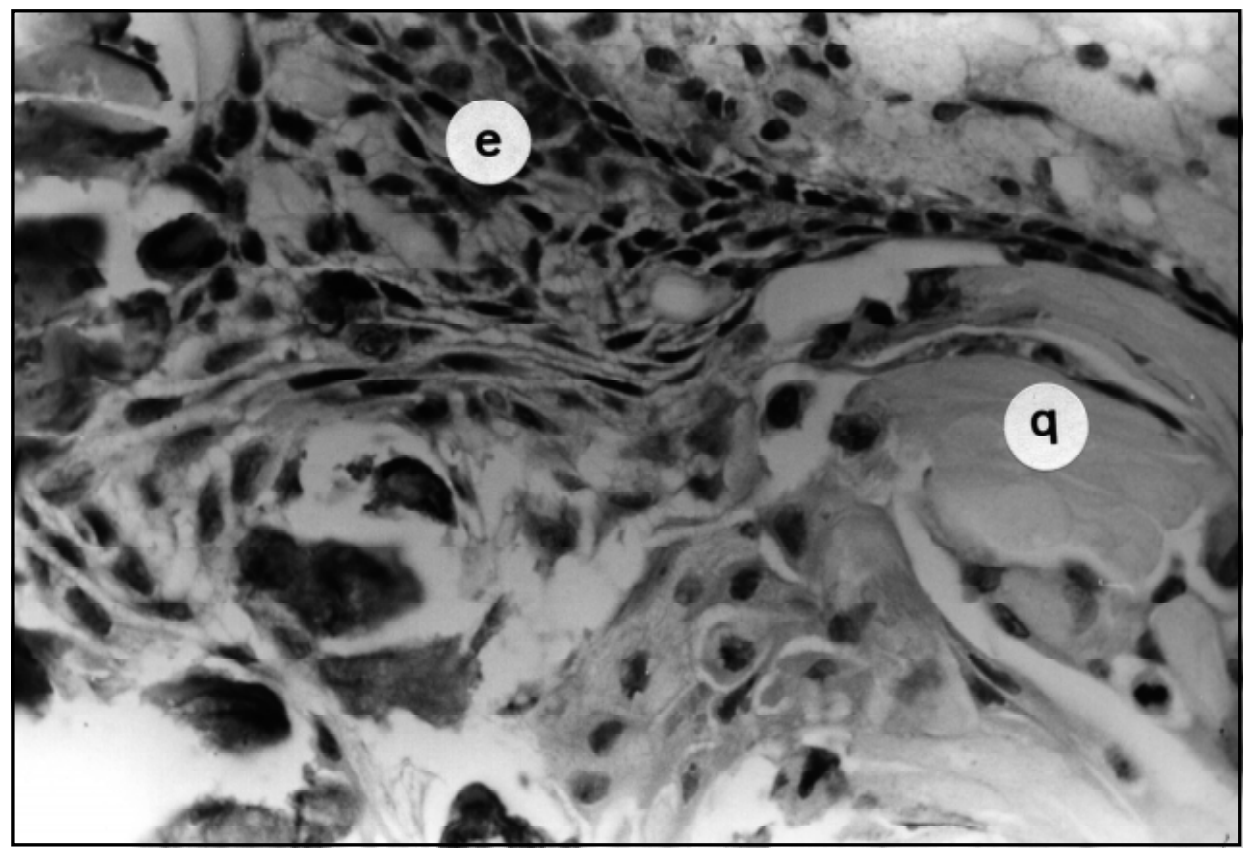

Fig. 8. Detalhe dos focos de queratinização (q) de craniofaringioma em meio a epitélio pavimentoso (e). HE x 400.

O tratamento dos craniofaringiomas é a ressecção cirúrgica total ${ }^{1-4,6,10}$, sendo que a associação de radioterapia nos casos em que a exérese completa não pôde ser realizada é controversa ${ }^{1-4,6,10}$. As taxas de recidiva e de sobrevida estão intrinsecamente relacionadas com a taxa de neoplasia macroscopicamente residual, bem como as dimensões tumorais, sendo que tumores com mais de $5 \mathrm{~cm}$ no momento do diagnóstico apresentam um prognóstico drasticamente pior ${ }^{1-4,6,10}$. Em nosso estudo, o único fator prognóstico identificado foi a extensão da ressecção cirúrgica, havendo taxas de recidiva de $36,4 \%$ nos casos submetidos à ressecção total e de 53,8\% dos submetidos à ressecção parcial.

\section{REFERÊNCIAS}

1. Kleihues P, Burger PC, Scheithauer BW. Histological typing of tumours of the central nervous system. World Health Organization international classification of tumours. 2Ed. Berlin:Springer, 1993.

2. Crotty TB, Scheithauer BW, Young WF Jr, et al.. Pappilary craniopharyngioma: a clinicopathological study of 48 cases. J Neurosurg, 1995;83:206-214.

3. Hetelekids S, Barnes PD, Tao ML, et al.. 20-year experience in childhood craniopharyngiomas. Int J Radiat Oncol Biol Phys, 1993;27:189-195.

4. Weiner K, Wisoff JH, Rosenberg ME, et al.. Craniopharyngiomas: a clinicopathological analysis of factors predictive of recurrence and functional outcome. Neurosurgery, 1994;35:1001-1010.

5. Bancroft JD, Stevens A. Theory and practice of histological techniques. 2Ed. New York: Churchill Livingstone, 1982.

6. Adamson TE, Wistler OD, Kleihues P, Yasargil MG. Correlation of clinical and pathological features in surgically treated craniopharyngiomas. J Neurosurg, 1990;73:12-17.

7. Giangaspero F, Burger PC, Osborne DR, Stein RB. Suprasellar pappilary squamous epithelioma ("pappilary craniopharyngioma"). Am J Surg Pathol, 1984;8:57-64.

8. Sanford RA. Craniopharyngioma: Results of survey of the American Society of Pediatric Neurosurgery. Pediatr Neurosurg, 1994;21(Suppl 1):39-43.

9. Harwood Nash DC. Neuroimaging of child craniopharyngiomas. Pediatr Neurosurg, 1994;21(Suppl 1):2-10.

10. Yasargil MG, Curcic M, Kis M, Siegenthaler G, Teddy PJ, Roth P. Total removal of craniopharyngiomas: approaches and long-term results in 144 patients. J Neurosurg, 1990;73: 3-11.

11. Tachibana O, Yamashina T, Yamashita J, Takabatake Y. Immunohistochemical expression of human chorionic gonadotropin and p-glycoprotein in human pituitary glands and craniopharyngiomas. J Neurosurg, 1994;80:79-84.

12. Szeifert GT, Pasztor E. Could craniopharyngiomas produce pituitary hormones? Neurol Res, 1993;15: 68-69.

13. Thapar K, Stefaneanu L, Kovacs K, et al.. Estrogen receptor gen expression in craniopharyngiomas: an in situ hybridization study. Neurosurgery, 1994;35:1012-1017. 